\title{
Rigorous derivation of the Darcy boundary condition on the porous wall
}

\author{
Eduard Marusic-Paloka ${ }^{1}$ \\ ${ }^{1}$ University of Zagreb
}

March 8, 2022

\begin{abstract}
Viscous flow through a reservoir with porous boundary is studied via rigorous asymptotic analysis. Under the assumption of periodicity of the pores, the effective boundary condition of the Darcy type is derived, using homogenization and boundary layer techniques. Further asymptotic analysis with respect to the porosity yields a recursive sequence of boundary value problems showing that the large pressure jump occurs on the boundary.
\end{abstract}

\section{Hosted file}

porous-boundary-Stokes5.pdf available at https://authorea.com/users/464091/articles/559058rigorous-derivation-of-the-darcy-boundary-condition-on-the-porous-wall 
figures/Fig1/Fig1-eps-converted-to.pdf 
figures/Fig2/Fig2-eps-converted-to.pdf 